\title{
Host Genetic Background and Gut Microbiota Contribute to Differential Metabolic Responses to Fructose Consumption in Mice
}

In Sook Ahn, ${ }^{1,8}$ Jennifer M. Lang, ${ }^{2,8}$ Christine Olson, ${ }^{1}$ Guanglin Zhang, ${ }^{1}$ Zhe Ying, ${ }^{1}$ Hyae Ran Byun, ${ }^{1}$ Graciel Diamante, ${ }^{1}$ Ingrid Cely, ${ }^{1}$ Jessica Ding, ${ }^{1}$ Ira Kurtz, ${ }^{3,4}$ Fernando GomezPinilla, ${ }^{1,5}$ Elaine Hsiao, ${ }^{1}$ Aldons J. Lusis, ${ }^{2}$ and Xia Yang ${ }^{1,4,6,7^{*}}$

${ }^{1}$ Department of Integrative Biology and Physiology, University of California, Los Angeles, CA

${ }^{2}$ Department of Medicine/Division of Cardiology, David Geffen School of Medicine, University of California, Los Angeles, CA

${ }^{3}$ Department of Medicine, Division of Nephrology, University of California, Los Angeles, CA

${ }^{4}$ Brain Research Institute, University of California, Los Angeles, CA

${ }^{5}$ Department of Neurosurgery, University of California, Los Angeles, CA 90095

${ }^{6}$ Molecular Biology Institute, University of California, Los Angeles, CA, USA

${ }^{7}$ Institute for Quantitative and Computational Biosciences, University of California, Los Angeles, CA, USA

${ }^{8}$ These authors contributed equally

\section{*Corresponding author:}

Xia Yang, Ph.D.

Department of Integrative Biology and Physiology, University of California, Los Angeles Los Angeles, CA 90095, USA

Tel: (310) 206-1812, Email: xyang123@ucla.edu

\section{Running title: Gut Microbiota in Fructose Response}




\begin{abstract}
Background: High fructose consumption induces disparate metabolic responses between mouse strains.

Objective: Our goal is to investigate the role of gut microbiota in the differential metabolic responses to fructose in genetically diverse mouse strains, namely, C57BL/6J (B6), DBA/2J (DBA), and FVB/NJ (FVB).
\end{abstract}

Methods: Eight-week old male mice of B6, DBA, and FVB strains were given $8 \%$ fructose in the drinking water for 12 weeks. Using 16S ribosomal RNA sequencing, the gut microbiota composition was analyzed from cecum and feces, and correlated with metabolic phenotypes and host gene expression in hypothalamus, liver, and adipose tissue to prioritize microbial taxa that may contribute to differential host fructose responses. Lastly, fecal transplant and Akkermansia muciniphila colonization experiments were conducted to test the causal role of gut microbiota in determining mouse strain-specific fructose responses.

Results: DBA mice consuming fructose demonstrated significant increases in body weight, adiposity, and glucose intolerance, which were accompanied by low baseline levels of Akkermansia, S24-7, and Turicibacter, compared to B6 and FVB mice which did not exhibit body mass and glycemic alterations. Additionally, fructose altered several microbial taxa that demonstrated strain-specific correlations with metabolic phenotypes and gene expression in host tissues. From the fecal microbiota transplant experiments between B6 and DBA, B6 microbes abrogated the fructose response in DBA mice by conferring resistance to weight and fat gain and glucose intolerance. Further, DBA mice receiving Akkermansia muciniphila, which was enriched in B6 and FVB, also mitigated fructose-induced metabolic dysfunctions. 
bioRxiv preprint doi: https://doi.org/10.1101/439786; this version posted May 14, 2019. The copyright holder for this preprint (which was not certified by peer review) is the author/funder. All rights reserved. No reuse allowed without permission.

Conclusions: Our findings support that differential microbiota composition between mouse strains is partially responsible for host metabolic sensitivity to fructose, and Akkermansia is one of the key responsible microbiota that confers resistance to fructose-induced dysregulation of adiposity and glycemic traits. 


\section{INTRODUCTION}

The drastic increase in fructose consumption for the past few decades has been paralleled by the rising prevalence of metabolic syndrome and diabetes $(1,2)$. Our recent systems nutrigenomics study unraveled the impact of fructose on transcriptome, epigenome and gene-gene interactions in the hypothalamus, which is the master regulatory center of metabolic control (3). Additionally, studies on the liver tissue have revealed fructose-induced alterations in genes involved in several aspects of systemic metabolism $(4,5)$. Interestingly, different mouse strains with genetic diversity demonstrate disparate metabolic responses to fructose consumption, a finding reproducible across multiple studies, including ours $(6,7)$. However, the causal mechanisms underlying the inter-individual differences in metabolic responses to fructose are yet to be elucidated.

The gut microbiome is emerging as an important modulator of metabolism, obesity, and other metabolic disorders (8). In addition, the dynamic nature and ease of manipulation have made the gut microbiome a suitable therapeutic target to mitigate metabolic syndrome $(9,10)$. Diet influences trillions of gut microorganisms as early as one day post dietary intervention (11), and the response to a high fat high sucrose diet among genetically diverse mouse strains is linked by the gut microbiome as a mediator between the genetic and phenotypic diversity (12). It is plausible that the gut microbiome also plays a role in determining inter-individual variability in metabolic responses to fructose consumption.

Dietary fructose is mostly absorbed in the small intestine, where fructose is also extensively metabolized into glucose and organic acids (13). However, excessive amount of fructose that is not absorbed in the small intestine due to malabsorption or high intake can undergo colonic bacterial fermentation, resulting in the production of metabolites including short-chain fatty acids (SCFA) (14). Fructose also has the potential to modify microbiota 
community and functionality. For instance, fructose metabolites can shape the gut environment and provide energy sources for gut microbiota (15), and fructose can suppress gut bacterial colonization by silencing a colonization factor in a commensal bacterium (16).

Here, we investigated gut microbiota as a potential causal link between fructose consumption and differential metabolic phenotypes in mice with diverse genetic backgrounds. We tested our paradigm in three mouse strains, namely C57BL/6J (B6), DBA/2J (DBA), and FVB/NJ (FVB), that had contrasting metabolic susceptibility to high caloric diets $(7,12)$. We found distinct gut microbiota composition accompanying disparate metabolic phenotypes in response to fructose consumption across strains. Furthermore, our results suggest a causal role of gut microbiota and pinpoint specific bacteria in determining metabolic sensitivity to fructose. These findings indicate that the treatment of metabolic syndrome would optimally be based on an individualized approach which takes into consideration the genetic and microbial background of each individual.

\section{METHODS}

\section{Animals and study design}

Seven-week old male mice (20-25g) from three inbred strains, namely B6, DBA, and FVB, were obtained from the Jackson Laboratory (Bar Harbor, ME, USA) and housed in a pathogen-free barrier facility with a 12-hour light/dark cycle at University of California, Los Angeles. Mice were fed a standard chow diet (Lab Rodent Diet 5001, LabDiet, St Louis, MO). After one week acclimation, mice from each strain were randomly divided into two groups (Figure 1). One group was provided with regular drinking water (control group, $n=8-10$ mice/strain) and the other group (fructose group, $\mathrm{n}=10-12 \mathrm{mice} / \mathrm{strain})$ was given $8 \%(\mathrm{w} / \mathrm{v})$ fructose $(3.75 \mathrm{kcal} / \mathrm{g}$ energy; 
NOW Real Food, Bloomingdale, IL) in the drinking water for 12 weeks ad libitum. The 8\% fructose water has an energy density lower than the US popular Coca Cola (11.1\% sugar; 7.2 $\mathrm{g} / 100 \mathrm{ml}$ of fructose) (17), and therefore is physiologically relevant. The measurement of food and drink intake, metabolic phenotypes, and transcriptomic studies of hypothalamus, liver, and mesenteric adipose tissues were performed as described in Zhang et al (6). Gut microbiota composition was analyzed from cecum and feces, as detailed below, and was correlated with metabolic phenotypes and host gene expression in individual tissues to prioritize microbial taxa that may contribute to differential host fructose responses. Lastly, fecal transplant and Akkermansia muciniphila colonization experiments were conducted to test the causal role of gut microbiota in determining mouse strain-specific fructose responses (Figure 1). Experimental protocols were approved by the Institutional Animal Care and Use Committee at the University of California, Los Angeles.

\section{Fecal and cecal sample collection}

Each group of mice were housed in 2-3 cages $(n=3-4$ mice/cage). Fecal samples were collected by gently restraining each mouse in a sterile empty cage, with tail lifted, to induced defecation. Feces were immediately placed into liquid nitrogen and stored at $-80{ }^{\circ} \mathrm{C}$ for microbiota sequencing. Upon completion of the 12-week fructose feeding experiment, cecal contents were collected during terminal tissue dissection.

\section{Microbiota analysis}

Starting from the onset of fructose intake, fecal samples from weeks 1, 2, 4, and 12 and cecal samples at week 12 were used for microbiota sequencing ( $\mathrm{n}=8$ mice/time point/group/strain). 
Microbial DNA was isolated from fecal or cecal samples using the MO BIO PowerSoil@-htp 96 Well Soil DNA Isolation Kit (MO BIO, Carlsbad, CA). The V4 region of the 16S rRNA gene was amplified with barcoded primers, and amplicons were sequenced using Illumina HiSeq 4000. 16S rRNA sequences were analyzed using QIIME as detailed in Supplementary Data (18). Briefly, microbial communities were visualized with PCoA based on the UniFrac distance measure (19) and summarized into relative abundance for each taxonomic level in QIIME. Categorical groups (treatment, time, strain) were confirmed to have similar multivariate homogeneity of group dispersions to allow them to be compared using the non-parametric PERMANOVA test with the adonis function (20). Microbial composition was analyzed at the phylum, family, and genus taxonomic levels using the Statistical Analysis of Metagenomic Profiles (STAMP) software (21). Taxa that differed between the fructose and control groups were identified using the White's non-parametric T-test (22), followed by Storey's false discovery rate (FDR) estimation (23). Linear discriminant analysis (LDA) effect size (LEfSe) was used to identify taxa that discriminated between the microbiota of three mouse strains using standard parameters $(\mathrm{p}<0.05$, LDA score $>2.0)(24)$.

\section{Correlation analysis between gut microbes and metabolic phenotypes or tissue}

\section{transcriptome}

Correlation analysis was performed between the relative abundance of microbiota with metabolic phenotypes including body weight, adiposity, and area under the curve for glucose intolerance $\left(\mathrm{AUC}_{\mathrm{glucose}}\right)$ as well as expression levels of host genes altered by fructose in hypothalamus, liver, and adipose tissue, termed "fructose signature genes" as detailed in Zhang et al (6). Correlation was assessed using Biweight midcorrelation (bicor), which is resistant to outliers (25). Statistical 
p-values were adjusted using the Benjamini-Hochberg approach and FDR $<0.05$ was considered significant. Fructose signature genes in individual metabolic tissues that showed significant correlation with fructose-responsive microbiota were classified for their biological functions, as detailed in Supplemental Data.

\section{Fecal transplant between B6 and DBA mice}

Six-week old B6 or DBA recipient mice were orally gavaged with a solution of vancomycin $(50 \mathrm{mg} / \mathrm{kg})$, neomycin $(100 \mathrm{mg} / \mathrm{kg})$, and metronidazole $(100 \mathrm{mg} / \mathrm{kg})$ twice daily for 7 days. Ampicillin (1 mg/ml) was provided ad libitum in drinking water. Antibiotics-treated mice were housed in sterile cages with sterile water and food throughout the experiment.

Fecal samples were freshly collected from donor B6 and DBA mice fed chow and regular drinking water, and suspended at $200 \mathrm{mg} / 5 \mathrm{ml}$ in pre-reduced PBS. Antibiotics-treated recipient B6 or DBA mice were colonized with donor microbiota by oral gavage of $150 \mu$ fecal suspension from the donors of the other strain for 4 weeks. After 1 week of fecal transplant, mice from each recipient strain were divided into two groups (water or fructose group). The fructose group mice were placed on $8 \%$ fructose drinking water for 12 weeks while the water group received drinking water ( $\mathrm{n}=8 \mathrm{mice} / \mathrm{group} / \mathrm{strain})$. Metabolic phenotypes such as body weight, adiposity, and glucose intolerance were measured (detailed in Supplemental Data).

\section{Akkermansia colonization in DBA mice}

A. muciniphila (ATCC BAA845) was cultured under anaerobic condition at $37^{\circ} \mathrm{C}$ in Brain Heart Infusion (BHI) media supplemented with $0.05 \%$ hog gastric mucin type III (Sigma Aldrich, Calsbad, CA). Cultures were authenticated by full-length 16S rDNA sequencing (Laragen, 
Culver City, CA). The freshly cultured A. muciniphila was pelleted and re-suspended at $5 \times 10^{9}$ $\mathrm{cfu} / \mathrm{ml}$ in pre-reduced PBS. Antibiotics-treated DBA mice were orally gavaged with $200 \mathrm{ul}$ bacterial suspension twice during the first 3 weeks, and once a week afterwards for 6 weeks. After 1 week of bacterial gavage, DBA mice were divided into two groups (water or fructose group). The fructose group mice were placed on $8 \%$ fructose drinking water for 8 weeks while the water group received drinking water ( $\mathrm{n}=8$ /group), and metabolic phenotypes were measured (detailed in Supplemental Data).

\section{Statistical analysis}

Differences in metabolic phenotypes between control and treatment groups were analyzed using the Student's t-test. Other statistical tests for individual analyses were described in the corresponding sections above.

\section{RESULTS}

\section{Strain-specific metabolic responses to fructose treatment}

As detailed in Zhang et al (6), B6, DBA, and FVB mice demonstrated striking differences in their metabolic responses to $8 \%$ fructose treatment for 12 weeks. Briefly, in the absence of significant differences in the overall energy intake between the fructose and control groups in each of the three mouse strains, DBA mice were more sensitive to fructose in terms of obesity and diabetes-related phenotypes including body weight, adiposity, and glucose intolerance. In contrast, B6 mice showed significant increases while FVB mice had decreases in plasma cholesterol levels. These results demonstrate strong inter-strain variability in fructose response in genetically divergent mice. 


\section{Overall effects of fructose on gut microbiota community}

Since gut microbiota is an important modulator of metabolic disorders (8), we examined the effects of fructose on gut microbiota in each mouse strain using $16 \mathrm{~S}$ rDNA sequencing. Sample distribution in the PCoA plot showed distinctive clusters determined by mouse strain for both the cecum (PERMANOVA pseudo-F $=5.16, \mathrm{p}=0.001)$ and the fecal samples (PERMANOVA pseudo-F $=12.56, \mathrm{p}=0.001)($ Figure $2 \mathrm{~A}, \mathrm{~B})$. For fecal microbiota, time was also a significant overall factor (PERMANOVA pseudo-F $=2.8, \mathrm{p}=0.001$ ), with week 12 separating from the earlier weeks across mouse strains (Figure 2C) and in individual mouse strains (Figure 2D-F). Gut microbiota composition was also influenced by fructose treatment based on mouse strain, time, and location of microbiota. Fecal microbiota differences were seen at 12 weeks (indicated by dotted line) between fructose and water groups in B6 (Figure 2G, pseudo-F = 11.702, $\mathrm{p}=0.001$ ) and DBA mice (Figure $2 \mathrm{H}, \mathrm{pseudo}-\mathrm{F}=3.878, \mathrm{p}=0.046$ ) but not in FVB (Figure 2I). In cecum, the fructose group showed separation from controls for the B6 strain (Figure 2J), although the statistical significance could not be analyzed by PERMANOVA due to different group dispersions (PERMANOVA assumes equal dispersion). Fructose treatment was a significant factor for microbiota composition in DBA (Figure $2 \mathrm{~K}$, pseudo-F $=4.357, \mathrm{p}=0.002$ ), but not for FVB (Figure 2L). Overall, consistent with the differential effects of fructose on metabolic phenotypes, DBA gut microbiota were sensitive to fructose treatment.

\section{Baseline differences in gut microbiota between mouse strains show correlation with host} metabolic phenotypes 
Differences in baseline microbial composition can drive distinct host responses to the same dietary manipulation (26,27). At the phylum level, DBA feces showed significantly higher Firmicutes/Bacteroidetes ratio $(1.78 \pm 0.17, \mathrm{p}<0.05)$ than $\mathrm{B} 6(0.35 \pm 0.24)$ and FVB $(0.90 \pm$ 0.09) (Supplementary Figure 1), agreeing with the known association of higher Firmicutes with obesity (28) and the increased adiposity in DBA. The microbial taxa that account for the greatest differences between mouse strains based on LEfSe included 21 cecal and 14 fecal microbial genera that were differentially enriched in each strain (Supplementary Figure 2A-D).

We reasoned that if any specific microbial taxon determines the differential fructose response between mouse strains, its abundance likely shows contrasting patterns between DBA and the other two strains. Compared to B6 and FVB, DBA mice were more enriched for Lactobacillus, unknown bacteria of order Clostridiales, and unknown bacteria of family Lachnospiraceae. On the other hand, DBA mice were least enriched for unknown bacteria of family S24-7, Akkermansia, and Turicibacter (Figure 3A-F). To understand the potential role of these microbial taxa in metabolic regulation, we correlated the abundance of the above taxa with adiposity gain across strains and found all but Turicibacter were positively or negatively correlated with adiposity gain (Figure 3G-K).

\section{Fructose-responsive microbiota and correlation with host metabolic phenotypes}

To explore differential microbial response to fructose in the three mouse strains, we analyzed the abundance of gut microbiota after 12 weeks of fructose treatment. In DBA cecum, family Erysipelotrichaceae and its two genera (Clostridum and an unknown genus), and Anaerostipes were all significantly decreased by fructose. Fructose also significantly increased cecal Bifidobacterium in FVB mice, while no cecal taxa were significantly changed in B6 mice. In 
feces, however, B6 microbiota was altered by fructose treatment the most (nine families and three genera), with significant decreases in five taxa belonging to phylum Firmicutes and significant increases in Verrucomicrobiaceae and its genus Akkermansia, which has anti-obesity and insulin sensitivity effects $(27,29,30)$, and S24-7 of phylum Bacteroidetes, which encompasses $\sim 50 \%$ of the microbiota community. In DBA feces, fructose altered the abundance of Rikenellaceae and Pseudomonadaceae, and their genera. These two families were also found to be fructose-responsive in B6 mice; however, the changes were more dramatic in DBA mice (Table 1, Supplementary Figure 3). No fecal microbial taxa were significantly altered by fructose in FVB mice.

We next correlated the abundance of these fructose-responsive taxa with metabolic phenotypes in control or fructose-treated mice. In DBA cecum, an unknown genus of Erysipelotrichaceae was negatively correlated with adiposity and $\mathrm{AUC}_{\text {glucose }}$ (Supplementary Figure 4). In DBA feces, unknown genus of Rikenellaceae showed no significant correlation with body weight, adiposity, or $\mathrm{AUC}_{\text {glucose }}$ in the water group, but negative correlated with body weight and adiposity and positively correlated with $\mathrm{AUC}_{\text {glucose }}$ in the fructose group (Figure 4AC). No phenotypic correlation was observed for the fructose-responsive taxa in B6 and FVB mice, which is not surprising given the weaker phenotypic alterations in these strains in response to fructose consumption.

\section{Correlation of fructose-responsive microbiota with fructose signature genes in host}

\section{metabolic tissues}

We next questioned whether the fructose-responsive gut microbiota could regulate host gene expression, which may indicate potential communications from the gut to other host metabolic 
tissues. To address this, we analyzed the correlation between the abundance of fructoseresponsive microbiota and the host fructose signature genes in liver, adipose tissue, and hypothalamus (6). We observed distinct correlation patterns in the three mouse strains: the B6 fructose-responsive taxa were correlated with only hypothalamic fructose signature genes, while the fructose-responsive taxa in DBA cecum or feces were correlated with only liver or adipose tissue signature genes, respectively (Table 2, Supplementary table 1).

In B6, Dehalobacterium was decreased by fructose treatment, and showed positive correlation with hypothalamic genes encoding the neurotransmitter transporter Slc6a3, a notch signaling component Nrarp, and an autophagy gene Atg3. Akkermansia was increased in response to fructose and correlated with several neurotransmitter related genes, including $O x t$ with insulin-like activities (31) and Th encoding tyrosine hydroxylase. In DBA cecum, both Anaerostipes and Clostridium were positively correlated with Cyp $8 b 1$ in liver, which is responsible for bile acid synthesis (32). In DBA feces, all fructose-responsive taxa were correlated with host signature genes of the adipose tissue, and these genes were involved in lipid metabolism, immune system, vesicle mediated transport, and cell communication. Adipose genes such as Abhd3, Msrl, Ccrl, Creb, and Fas were correlated with both Rikenellaceae and Pseudomonadaceae and their genera. Taken together, these correlations suggest that gut microbiota may interact with host genes in a mouse strain- and tissue-specific manner in response to fructose (Table 2, Supplementary table 1).

\section{Alteration of gut microbiota modulates fructose response}

Since B6 and DBA mice showed disparate metabolic responses to fructose, we tested whether B6 microbiota confers resistance and DBA microbiota confers vulnerability by transplanting B6 
feces to antibiotics-treated DBA mice and vice versa (Figure 5A). Using16S rDNA sequencing post fecal transplant, we confirmed that the recipient mice experienced shifts in the gut microbiota throughout the fecal transplant experiments (Supplementary Figure 5). DBA mice receiving $\mathrm{B} 6$ feces $[\mathrm{DBA}(\mathrm{B} 6)]$ became resistant to body weight gain, fat mass gain, and glucose intolerance in spite of the fructose treatment, recapitulating the resistance phenotype of B6 mice. In contrast, B6 mice receiving DBA feces [B6(DBA)] stayed lean and glucose tolerance, suggesting that DBA microbiota failed to induce fructose sensitivity in B6 (Figure 5).

The above results from the fecal transplant experiment supported a causal role of B6 microbiota in conferring fructose resistance in DBA. We next focused on prioritizing the potential microbes in B6 that may determine the fructose resistance phenotype. Akkermansia was found to be a highly plausible candidate to explain the dampened response to fructose in B6 for the following reasons. First, Akkermansia has been previously demonstrated to carry anti-obesity and insulin-sensitivity effects $(27,29,30)$. Beneficial effect of Akkermansia was also previously observed in mice fed a high-fat/high-sucrose diet (33). Secondly, it was depleted in the vulnerable strain DBA but was highly abundant in both resistant strains, B6 and FVB (Figure 3). Thirdly, fructose treatment caused an increase in Akkermansia in B6 mice (Table 1, Supplementary Figure 3). Lastly, Akkermansia abundance in B6 is significantly correlated with a large number of hypothalamic genes such as $O x t$ and $T h$ that regulate metabolism (Table 2, Supplementary Table 1).

To test the role of Akkermansia in protecting against fructose-induced metabolic dysregulation as predicted above, we gavaged antibiotic-treated DBA mice with Akkermansia muciniphila while having them consume fructose for 8 weeks. Indeed, DBA mice receiving Akkermansia became resistant to body weight and fat mass gain and glucose intolerance in spite 
of the fructose treatment (Figure 6). These results support that Akkermansia confers resistance to fructose-mediated metabolic dysregulation.

\section{DISCUSSION}

Our studies showed that three mouse strains representing a range of genetic diversity differed in their metabolic and transcriptomic responses to high fructose treatment (6). Since gut microbiota is an important modulator of metabolic capacity (8), here we tested the hypothesis that the disparate responses among mouse strains were at least partially driven by the gut microbiota. Our 16S rDNA sequencing analysis revealed that baseline microbiota composition and its response to fructose varied by mouse strains. Fecal transplant data supports that B6 mice carry microbiota that confers resistance to fructose-induced metabolic dysfunctions. We next prioritized Akkermansia as a candidate taxon to explain the dampened response to fructose in B6 mice, which are enriched with Akkermansia, in comparison to DBA mice, which show depletion of Akkermansia. Indeed, gavaging A. muciniphila to DBA mice mitigated fructose-induced obesity and glucose intolerance. These results support a causal role of gut microbiota in determining the differential metabolic responses to fructose among genetically diverse mouse strains.

As initial colonizing microbial species are important for establishing a favorable environment for bacterial growth in a particular context (34), differences in baseline microbial composition between mouse strains can lead to variations in the metabolic processes of individuals in response to diet (26). We found that Akkermancia, Turicibacter, and S-24 were almost depleted in DBA mice but were abundant in B6 and FVB mice, whereas Lactobacillus and Lachnospiraceae were at higher baseline levels in DBA mice. Most of these bacteria were correlated with adiposity (Figure 3). Previously, Akkermansia has been associated with obesity 
resistance and improved metabolic parameters in humans and mice, and beneficial effects of dietary interventions have been associated with the higher abundance of Akkermansia at baseline (27,30). S24-7 has protective association against diabetes whereas Lachnospiraceae promotes pathogenesis of diabetes in NOD mice (35). The observed lack of Akkermansia and S-24, along with the higher abundance in Lachnospiraceae in DBA mice, agrees with the vulnerability of DBA mice to fructose-induced metabolic dysregulation. Therefore, these bacterial taxa that differ significantly at baseline between strains have the potential to regulate the differential response to fructose treatment.

In addition to differences in baseline microbes that can explain inter-mouse strain variability, bacteria altered by fructose may also play a role in the variability in metabolic responses to fructose. It has been suggested that fructose shifts the gut microbiota and leads to a westernized microbiome acquisition with altered metabolic capacity, resulting in development of obesity or metabolic disorders (36). In our study, we found various microbes with significantly altered abundances in DBA and B6 but not FVB, which may relate to their differential sensitivity to fructose. DBA cecal Erypsipelotricaceae and Anaerostipes decreased in abundance upon fructose consumption (Table 1, Supplementary Figure 3, Figure4), and they are known as butyrate-producing bacteria $(37,38)$. Butyrate promotes the intestinal barrier development, and decreased butyrate production can increase intestinal permeability (39). In both the B6 and DBA feces, we also detected decreased abundance of Rikenellaceae and increased abundance of Pseudomonadaceae following fructose treatment (Table 1). Increases in Pseudomonadaceae and Pseudomonas were previously found in obese individuals with higher insulin resistance (40). Therefore, despite the numerous differences between B6 and DBA, there were shared microbial changes in response to fructose consumption. The weaker phenotypic responses in B6 can be a 
result of compensatory balancing effects of higher abundances of beneficial bacteria such as Akkermansia and S24-7 in B6.

We detected more fructose-responsive microbiota in B6 mice than in DBA and FVB mice (Table 1), and, surprisingly, most of these B6 taxa were associated with the hypothalamic genes responsive to fructose (Table 2, Supplementary table 1). A hypothalamic gene correlating with Dehalobacterium in B6 is Nrarp (positively correlated), which encodes an intracellular component of the Notch signaling pathway and regulates differentiation of mouse hypothalamic arcuate neurons responsible for feeding and energy balance. Dysregulation of this homeostatic mediator underlies diseases ranging from growth failure to obesity (41). Akkermansia was high in B6 at baseline and was further increased by fructose treatment, and was negatively correlated with gene $T h$. Hypothalamic arcuate nucleus TH neurons play a role in energy homeostasis, and silencing of TH neurons reduces body weight (42). Our previous study (3) reported fructose as a powerful inducer of genomic and epigenomic variability with the capacity to reorganize gene networks critical for central metabolic regulation and neuronal processes in the hypothalamus. The current study suggests that fructose-induced changes in hypothalamic gene expression in B6 mice could be partly via fructose-responsive gut microbiota.

In contrast to B6, fructose responsive microbiota in DBA was more associated with lipid and inflammatory genes in the adipose tissue (Table 2, Supplementary table 1). Abhd3 is a crucial factor for insulin resistance in adipose tissue (43); Sema3e contributes to inflammation and insulin resistance in obese mice (44); Msrl is macrophage scavenger receptor 1, provides a protection from excessive insulin resistance in obese mice (45); Creb promotes expression of transcriptional factors of adipogenesis and insulin resistance in obesity (46); Fas contributes to adipose tissue inflammation, hepatic steatosis, and insulin resistance induced by obesity (47). 
Therefore, these adipose-tissue genes correlating with fructose-responsive bacteria in DBA are relevant to the increased adiposity and compromised insulin sensitivity seen in DBA mice. However, we acknowledge that the correlative relationship observed here does not directly imply causation, and future experiments are needed to directly test the causal role of the genes as well as the bacteria implicated.

Our fecal transplant study support that B6 mice carry gut microbes that confer resistance to fructose-induced metabolic syndrome, and that Akkermansia partially mediates this protective effect. Given the recognized therapeutic potential of modulating the gut microbiota (10), probiotic treatment with Akkermansia may represent a viable approach to mitigate fructoseinduced metabolic abnormalities. In addition to Akkermansia, other microbes may also play a role in modulating the differential fructose response between individuals. Future efforts testing the pathogenic or protective role of other individual bacteria revealed, such as Lactobacillus, Turicibacter, or Pseudomonas, are warranted.

In summary, our multi-strain, multi-omics (gut microbiome, transcriptome, and phenome) integrative studies of inter-individual variability in fructose-induced metabolic syndrome established a causal role of gut microbiota in modulating host metabolic and tissue-specific transcriptomic responses to fructose, a significant metabolic risk in modern societies. Importantly, our study identified key microbial species that may serve as preventative or therapeutic targets for metabolic syndrome. By exploring gut-host interactions, our studies also open numerous hypotheses regarding specific microbiota-host interactions to be further elucidated in the future. 


\section{ACKNOWLEDGEMENT}

The authors thank Dr. Richard C. Davis for assistance of oral gavage and fecal transplant, Dr. Yuqi Zhao and Dr. Zeyneb Kurt for the assistance with data analysis, and Dr. Sana Majid for helping with animal experiments.

I.S.A. and J.M.L conducted the main experiments and analyses and wrote the manuscript. X.Y., A.J.L., E.H., F.G-P., J.M.L., I.S.A., I.K. and C.O. designed the experiment and revised manuscript. H.R.B., C.O., Z.Y., G. D., I. C., J. D. contributed to animal experiments. J.M.L. analyzed microbiome data. G.Z collected transcriptome data. X.Y. is the guarantor of this work and, as such, full access of all the data in the study and takes responsibility for the integrity of the data and the accuracy of the data analysis.

\section{FUNDING}

X.Y. and F.G-P. are funded by NIH DK104363 and NS103088. J.M.L. was supported by NIH

T32 DK007789. G.D. was supported by NIEHS NIH T32ES015457. I.K. is supported in part by DK077162, the Allan Smidt Charitable Fund, and the Factor Family Foundation Chair in Nephrology. A.J.L is supported by NIH HL28481 and HL30568.

\section{DUALITY OF INTEREST}

There are no potential conflicts of interest relevant to this article. 


\section{REFERENCES}

1. Basciano H, Federico L, Adeli K. Fructose, insulin resistance, and metabolic dyslipidemia. Nutr Metab 2005;2(5):1-14.

2. Stanhope KL, Schwarz JM, Keim NL, Griffen SC, Bremer AA, Graham JL,Hatcher B, Cox CL, Dyachenko A, Zhang W, et al. Consuming fructose-sweetened, not glucosesweetened, beverages increase visceral adiposity and lipids and decrease insulin sensitivity in overweight/obese men. J Clin Invest 2009;1334(5):1322-34.

3. Meng Q, Ying Z, Noble E, Zhao Y, Agrawal R, Mikhail A, Zhuang Y, Tyagi E, Zhang Q, Lee JH, et al. Systems Nutrigenomics Reveals Brain Gene Networks Linking Metabolic and Brain Disorders. EBioMedicine 2016;7:157-66.

4. Koo H, Wallig MA, Hong B, Nara TY, Cho BHS, Nakamura MT. Dietary fructose induces a wide range of genes with distinct shift in carbohydrate and lipid metabolism in fed and fasted rat liver. Biochim Biophys Acta 2008;1782:341-8.

5. Li Z, Xiong C, Mo S, Tian H, Yu M, Mao T, Chen Q, Luo H, Li Q, Lu J, et al. Comprehensive transcriptome analyses of the fructose-fed syrian golden hamster liver provides novel insights into lipid metabolism. PLoS One 2016;11(9):1-19.

6. Zhang G, Byun HR, Ying Z, Blencowe M, Zhao Y, Hong J, Shu L, Krishnan KC, GomezPinilla F, Yang X. Differential metabolic and multi-tissue transcriptomic responses to fructose consumption among genetically diverse mice. bioRxiv 2018; doi: https://doi.org/10.1101/439562

7. J. Glendinning, L. Breinager, E. Kyrillou, K. Lacuna, R. Rocha AS. Differential effects of sucrose and fructose on dietary obesity in four mouse strains. Physiol Behav 2010;101(3):331-43.

8. Turnbaugh PJ, Ley RE, Mahowald M a, Magrini V, Mardis ER, Gordon JI. An obesityassociated gut microbiome with increased capacity for energy harvest. Nature 2006;444(7122):1027-31.

9. Yadav H, Jain S, Sinha PR. Antidiabetic effect of probiotic dahi containing Lactobacillus acidophilus and Lactobacillus casei in high fructose fed rats. Nutrition 2007;23(1):62-8.

10. Fändriks L. Roles of the gut in the metabolic syndrome: an overview. J Intern Med 2016;1-18.

11. David LA, Maurice CF, Carmody RN, Gootenberg DB, Button JE, Wolfe BE, et al. Diet rapidly and reproducibly alters the human gut microbiome. Nature 2014;505(7484):55963.

12. Parks BW, Nam E, Org E, Kostem E, Norheim F, Hui ST, Pan C, Civelek M, Rau CD, Bennett, et al. Genetic control of obesity and gut microbiota composition in response to high-fat, high-sucrose diet in mice. Cell Metab 2013;17(1):141-52.

13. Jang C, Hui S, Lu W, Cowan AJ, Morscher RJ, Lee G, Liu W, Tesz GJ, Birnbaum MJ, Rabinowitz JD. The Small Intestine Converts Dietary Fructose into Glucose and Organic Acids. Cell Metab 2018;27(2):351-61.

14. Oh JH, Alexander LM, Pan M, Schueler KL, Keller MP,Attie AD, Walter J, Pijkeren J 
Van, et al. Dietary Fructose and Microbiota-Derived Short- Chain Fatty Acids Promote Bacteriophage Production in the Gut Symbiont Lactobacillus reuteri Article Dietary Fructose and Microbiota-Derived Short-Chain Fatty Acids Promote Bacteriophage Production in the Gut. Cell Host Microbe 2019;1-12.

15. Ríos-Covián D, Ruas-Madiedo P, Margolles A, Gueimonde M, De los Reyes-Gavilán CG, Salazar N. Intestinal short chain fatty acids and their link with diet and human health. Front Microbiol 2016;7(185):1-9.

16. Townsend GE, Han W, Schwalm ND, Raghavan V, Barry NA. Dietary sugar silences a colonization factor in a mammalian gut symbiont. PNAS 2019;116(1):233-8.

17. Ventura EE, Davis JN, Goran MI. Sugar content of popular sweetened beverages based on objective laboratory analysis: focus on fructose content. Obesity 2011;19(4):868-74.

18. Caporaso JG, Kuczynski J, Stombaugh J, Bittinger K, Bushman FD, Costello EK, Fierer N, Peña AG, Goodrich JK, Gordon JI, et al. QIIME allows analysis of high- throughput community sequencing data. Nat Methods 2010;7(5):335-6.

19. Lozupone C, Knight R. UniFrac : a New Phylogenetic Method for Comparing Microbial Communities 2005;71(12):8228-35.

20. Jari Oksanen, F. Guillaume Blanchet, Michael Friendly, Roeland Kindt, et al. vegan: Community Ecology Package. at <http://cran.r-project.org/package=vegan>. 2015

21. Parks DH, Tyson GW, Hugenholtz P, Beiko RG. STAMP : statistical analysis of taxonomic and functional profiles. Bioinformatics 2014;30(21):3123-4.

22. White JR, Nagarajan N, Pop M. Statistical methods for detecting differentially abundant features in clinical metagenomic samples. PLoS Comput Biol 2009;5(4):1-11.

23. Storey JD. Strong control, conservative point estimation and simultaneous conservative consistency of false discovery rates : a unified approach. J. R. Stat. Soc 2004;66(1):187205.

24. Segata N, Izard J, Waldron L, Gevers D, Miropolsky L, Garrett WS, Huttenhower C. Metagenomic biomarker discovery and explanation. Genome Biol 2011;12:1-18.

25. Zheng CH, Yuan L, Sha W, Sun ZL. Gene differential coexpression analysis based on biweight correlation and maximum clique. BMC Bioinform 2014;15(15):1-7.

26. Kootte RS, Levin E, Salojärvi J, Smits LP, Hartstra A V., Udayappan SD, Hermes G, Bouer KE, Koopen AM, Holst JJ, et al. Improvement of insulin sensitivity after lean donor feces in metabolic syndrome is driven by baseline intestinal microbiota composition. Cell Metab 2017;26(4):611-9.

27. Dao MC, Everard A, Aron-Wisnewsky J, Sokolovska N, Prifti E, Verger EO, Kayser BD, Levenez F, Chilloux J, Hoyles L, et al. Akkermansia muciniphila and improved metabolic health during a dietary intervention in obesity: relationship with gut microbiome richness and ecology. Gut 2016;65(3):426-36.

28. Ley RE, Backhed F, Turnbaugh P, Lozupone CA, Knight RD, Gordon JI. Obesity alters gut microbial ecology. Proc Natl Acad Sci 2005;102(31):11070-5.

29. Derrien M, Van Baarlen P, Hooiveld G, Norin E, Müller M, de Vos WM. Modulation of mucosal immune response, tolerance, and proliferation in mice colonized by the mucin- 
degrader Akkermansia muciniphila. Front Microbiol 2011;2(166):1-14.

30. Everard A, Belzer C, Geurts L, Ouwerkerk JP, Druart C, Bindels LB, Guiot Y, Derrien M, Muccioli GG, Delzenne NM, et al. Cross-talk between Akkermansia muciniphila and intestinal epithelium controls diet-induced obesity. Proc Nati Acad Sci 2013;110(22):9066-71.

31. Gimpl G, Fahrenholz F, Gene C. The Oxytocin Receptor System : Structure, Function, and Regulation. Physiol Rev 2001;81(2):629-84.

32. Wahlström A, Sayin SI, Marschall HU, Bäckhed F. Intestinal crosstalk between bile acids and microbiota and its impact on host metabolism. Cell Metab 2016;24(1):41-50.

33. Org E, Parks BW, Joo JWJ, Emert B, Schwartzman W, Kang EY, Mehrabian M, Pan C, Knight R, Gunsalus R, et al. Genetic and environmental control of host-gut microbiota interactions. Genome Res 2015;25(10):1558-69.

34. Jost T, Lacroix C, Braegger CP, Chassard C. New insights in gut microbiota establishment in healthy breast fed neonates. PLoS One 2012;7(8):e44595.

35. Krych, Nielsen DS, Hansen a. K, Hansen CHF. Gut microbial markers are associated with diabetes onset, regulatory imbalance, and IFN-r level in NOD Mice. Gut Microbes 2015;6(2):101-9.

36. Payne AN, Chassard C, Lacroix C. Gut microbial adaptation to dietary consumption of fructose, artificial sweeteners and sugar alcohols: Implications for host-microbe interactions contributing to obesity. Obes Rev 2012;13(9):799-809.

37. Estaki M, Pither J, Baumeister P, Little JP, Gill SK, Ghosh S, Ahmadi-Vand Z, Marsden $\mathrm{KR}$, Gibson DL. Cardiorespiratory fitness as a predictor of intestinal microbial diversity and distinct metagenomic functions. Microbiome 2016;4(42):1-13.

38. Levine UY, Looft T, Allen HK, Stanton TB. Butyrate-producing bacteria, including mucin degraders, from the swine intestinal tract. Appl Environ Microbiol 2013;79(12):3879-81.

39. Peng L, Li Z-R, Green RS, Holzman IR, Lin J. Butyrate Enhances the Intestinal Barrier by Facilitating Tight Junction Assembly via Activation of AMP-Activated Protein Kinase in Caco-2 Cell Monolayers. J Nutr 2009;139:1619-25.

40. Moreno-Indias I, Sánchez-Alcoholado L, García-Fuentes E, Cardona F, Queipo-Ortuño MI, Tinahones FJ. Insulin resistance is associated with specific gut microbiota in appendix samples from morbidly obese patients. Am J Transl Res 2016;8(12):5672-84.

41. Lamar E, Deblandre G, Wettstein D, Gawantka V, Pollet N, Niehrs C, Kintner C. Nrarp is a novel intracellular component of the Notch signaling pathway. Genes Dev 2001;15:1885-99.

42. Zhang X, Van Den Pol AN. Hypothalamic arcuate nucleus tyrosine hydroxylase neurons play orexigenic role in energy homeostasis. Nat Neurosci 2016;19(10):1341-7.

43. Xia W, Pessentheiner AR, Hofer DC, Amor M, Schreiber R, Schoiswohl G, Eichmann TO, Walenta E, Itariu B, Prager G, et al. Loss of ABHD15 Impairs the Anti-lipolytic Action of Insulin by Altering PDE3B Stability and Contributes to Insulin Resistance. Cell Rep 2018;23:1948-61. 
44. Shimizu I, Yoshida Y, Moriya J, Nojima A, Uemura A, Kobayashi Y, Minomino T. Semaphorin3E-induced inflammation contributes to insulin resistance in dietary obesity. Cell Metab 2013;18(4):491-504.

45. Cavallari, J. F., Anhe, F. F., Foley , K. P., Denou E., Chan R. W., Bowdish D. M. E., Schertzer J. D. Targeting macrophage scavenger receptor 1 promotes insulin resistance in obese male mice, Physiol Rep 2018; (22):1-11.

46. Qi L, Saberi M, Zmuda E, Wang Y, Altarejos J, Zhang X, Dentin R, Hedrick S, Bandyopadhyay G, Hai T, et al. Adipocyte CREB Promote insulin resistance in Obesity. Cell Metab 2010;9(3):277-86.

47. Wueest S, Rapold RA, Schumann DM, Rytka JM, Schildknecht A, Nov O, Chervonsky AV, Rudich A, Schoenle EJ, Donath MY, et al. Deletion of Fas in adipocytes relieves adipose tissue inflammation and hepatic manifestations of obesity in mice. J Clin Invest 2010;120(1):191-202. 
Table 1. Differential abundance of fructose-responsive microbiota in cecal and fecal samples of B6, DBA, and FVB.

\begin{tabular}{|c|c|c|c|c|c|}
\hline \multirow{2}{*}{$\begin{array}{l}\text { Mouse } \\
\text { strain }\end{array}$} & \multirow{2}{*}{$\begin{array}{l}\text { Micro- } \\
\text { biota } \\
\text { Source }\end{array}$} & \multirow[t]{2}{*}{ Fructose-responsive microbiota } & \multicolumn{2}{|c|}{ Relative abundance $(\%)($ Mean \pm SD $)$} & \multirow[t]{2}{*}{ FDR } \\
\hline & & & Fructose & Water & \\
\hline$\overline{\mathrm{DBA}}$ & Cecum & Erysipelotrichaceae & $0.007 \pm 0.003$ & $0.127 \pm 0.073$ & 0.038 \\
\hline \multirow[t]{9}{*}{ B6 } & Feces & Rikenellaceae & $11.600 \pm 2.861$ & $18.700 \pm 5.271$ & 0.043 \\
\hline & & S24-7 & $59.340 \pm 4.534$ & $49.890 \pm 5.544$ & 0.030 \\
\hline & & Dehalobacteriaceae & $0.030 \pm 0.008$ & $0.050 \pm 0.014$ & 0.020 \\
\hline & & Lachnospiraceae & $3.560 \pm 1.194$ & $6.030 \pm 1.358$ & 0.030 \\
\hline & & Mogibacteriaceae & $0.020 \pm 0.005$ & $0.060 \pm 0.020$ & 0.020 \\
\hline & & Ruminococcaceae & $2.280 \pm 0.454$ & $3.230 \pm 0.589$ & 0.032 \\
\hline & & Turicibacteraceae & $0.640 \pm 0.290$ & $1.840 \pm 0.763$ & 0.030 \\
\hline & & Pseudomonadaceae & $0.020 \pm 0.012$ & $0.006 \pm 0.002$ & 0.043 \\
\hline & & Verrucomicrobiaceae & $4.930 \pm 1.925$ & $0.680 \pm 0.475$ & 0.002 \\
\hline \multirow[t]{2}{*}{ DBA } & Feces & Rikenellaceae & $6.500 \pm 3.037$ & $17.370 \pm 2.776$ & 0.026 \\
\hline & & Pseudomonadaceae & $0.200 \pm 0.169$ & $0.006 \pm 0.003$ & 0.023 \\
\hline \multirow[t]{3}{*}{ DBA } & Cecum & Erysipelotrichaceae (Unknown genus) & $0.0004 \pm 0.0005$ & $0.106 \pm 0.061$ & 0.024 \\
\hline & & Erysipelotrichaceae Clostridium & $0.0001 \pm 0.003$ & $0.011 \pm 0.008$ & 0.001 \\
\hline & & Lachnospiraceae Anaerostipes & $0.004 \pm 0.005$ & $0.160 \pm 0.061$ & 0.001 \\
\hline FVB & Cecum & Bifidobacteriaceae Bifidobacterium & $0.099 \pm 0.072$ & $0.006 \pm 0.066$ & 0.049 \\
\hline \multirow[t]{3}{*}{ B6 } & Feces & Dehalobacteriaceae Dehalobacterium & $0.030 \pm 0.008$ & $0.050 \pm 0.014$ & 0.032 \\
\hline & & Mogibacteriaceae (Unknown genus) & $0.020 \pm 0.005$ & $0.060 \pm 0.020$ & 0.032 \\
\hline & & Verrucomicrobiaceae Akkermansia & $4.930 \pm 1.925$ & $0.678 \pm 0.475$ & 0.004 \\
\hline \multirow[t]{3}{*}{ DBA } & Feces & Rikenellaceae (Unknown genus) & $6.500 \pm 3.036$ & $17.380 \pm 2.776$ & 0.023 \\
\hline & & Pseudomonadaceae (Unknown genus) & $0.060 \pm 0.069$ & $0.001 \pm 0.002$ & 0.026 \\
\hline & & Pseudomonadaceae Pseudomonas & $0.140 \pm 0.170$ & $0.005 \pm 0.003$ & 0.026 \\
\hline
\end{tabular}

Taxa within an individual mouse strain at 12 weeks of the study were compared between the fructose and water treatment using the White's non-parametric T-test followed by Storey's false discovery rate (FDR) estimation. Taxa in family or genus level are capitalized or italicized, respectively. Sample size $n=8 /$ group/strain. 
Table 2. Correlation between fructose-responsive microbiota and host fructose signature genes in three metabolic tissues in individual mouse strains.

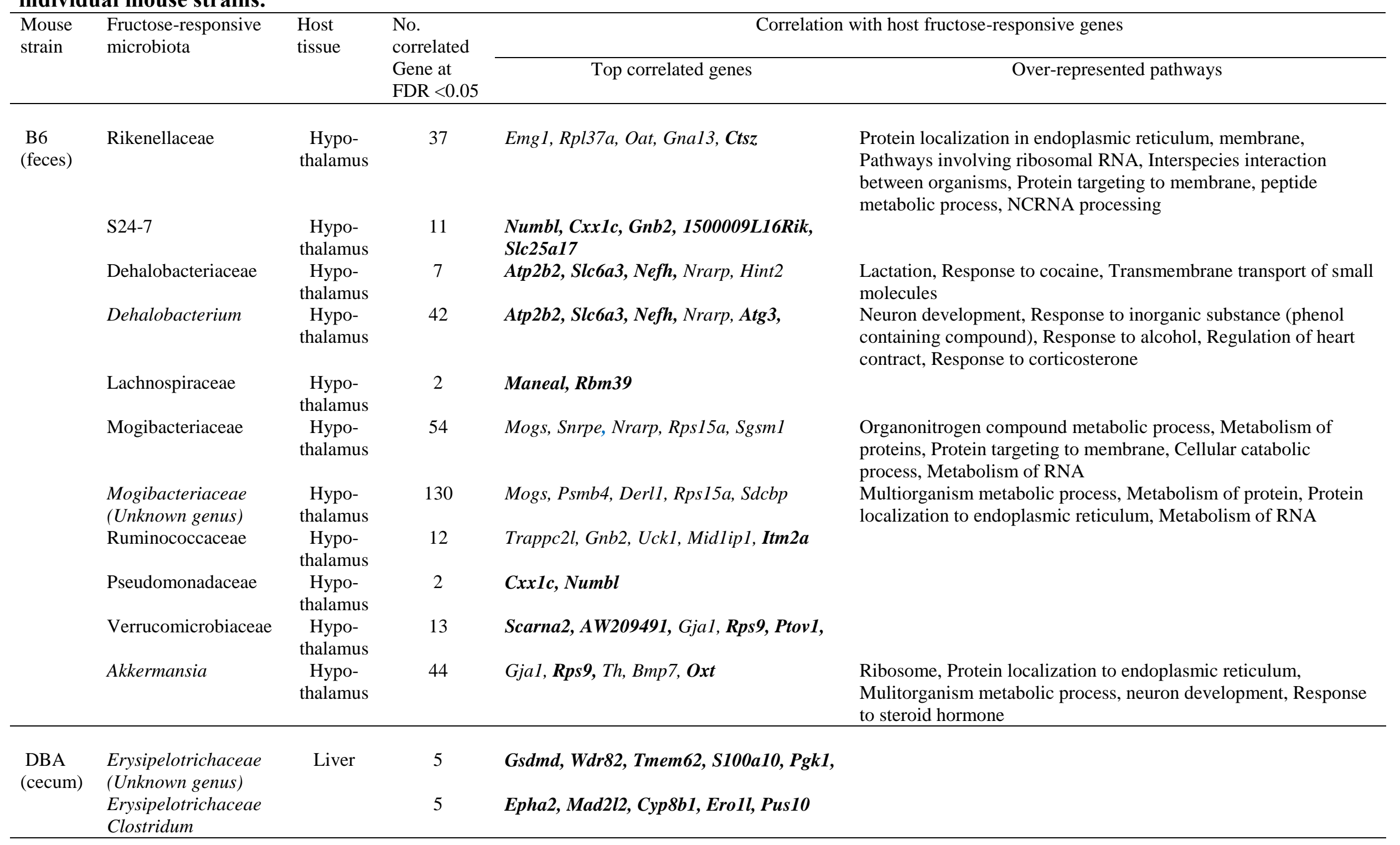




\begin{tabular}{|c|c|c|c|c|c|}
\hline & $\begin{array}{l}\text { Lachnospiraceae } \\
\text { Anaerostipes }\end{array}$ & Liver & 4 & Rogdi, Cyp8b1, Mad2l2, Baiap2 & \\
\hline \multirow[t]{5}{*}{$\begin{array}{l}\text { DBA } \\
\text { (feces) }\end{array}$} & Rikenellaceae & Adipose & 447 & Ccrl, Sema3e, Msr 1, Abhd3, Fas, & $\begin{array}{l}\text { Response to small molecule (lipid, cytokines, bacterial origin } \\
\text { molecule), Lipid metabolic process, Immune system process, } \\
\text { Vesicle mediated transport. Cell communication }\end{array}$ \\
\hline & $\begin{array}{l}\text { Rikenellaceae } \\
\text { (Unknown genus) }\end{array}$ & Adipose & 432 & Ccrl, Apobec1, Sema3e, Creb1, Fas & $\begin{array}{l}\text { Response to small molecule (organic compound, lipid, cytokines, } \\
\text { bacterial origin molecule), Lipid metabolic process, Immune } \\
\text { system process, Vesicle mediated transport, Cell communication }\end{array}$ \\
\hline & Pseudomonadaceae & Adipose & 77 & Sfrp4, Msr1, Sema3e, Abhd3, Fas & $\begin{array}{l}\text { Cell differentiation and cell death, Regulation of lipid metabolic } \\
\text { process, Autophagy, Response to stress, Regulation of lipid } \\
\text { metabolism }\end{array}$ \\
\hline & $\begin{array}{l}\text { Pseudomonadaceae } \\
\text { (Unknown genus) }\end{array}$ & Adipose & 209 & Creb1, Fabp3, Msr1, Sema3e, Fas & $\begin{array}{l}\text { RNA processing, Response to organic molecules, lipid, Response } \\
\text { to stimulus (bacterium), Catabolic process, Immune system } \\
\text { process }\end{array}$ \\
\hline & Pseudomonas & Adipose & 43 & $\begin{array}{l}\text { Ppp1r13b, Jmjd1c, Msr1, Slc25a10, } \\
\text { Fabp3 }\end{array}$ & \\
\hline
\end{tabular}

Fructose-responsive genes which were positively or negatively correlated with microbiota were indicated in bold or non-bold font, respectively. Top five correlated genes were picked from the genes with statistically higher significance which belongs to major gene sets analyzed using GSEA software. Correlation was analyzed using Biweight midcorrelation with FDR adjustment using the

Benjamini-Hochberg procedure. Sample size $n=4-6$ animals/group/strain with matching gut microbiota and gene expression data. Full lists of correlated genes and pathways are in Supplementary Table 1. 


\section{Figure legends}

Figure 1. Overall study design. Three mouse strains, C57BL/6J (B6), DBA/2J (DBA), FVB/NJ (FVB), were used to study the differential metabolic responses to fructose treatment. We treated mice with $8 \%$ fructose water for 12 weeks and collected metabolic phenotypes, microbiome data, and transcriptome data from host individual tissues (hypothalamus, liver, and mesenteric adipose tissue). The gut microbiota showing differential baseline levels between mouse strains as well as fructose-responsive microbiota were correlated with metabolic phenotypes and fructose signature genes in individual tissues to prioritize microbial species associated with host responses. The causal role of gut microbiota was validated by fecal transplant between B6 mice (fructose resistant) and DBA mice (fructose sensitive). Finally, Akkermansia was inoculated to DBA mice to determine its causal role in mitigating the fructose response in DBA mice.

\section{Figure 2. Clustering of cecal and fecal microbiota of three mouse strains with fructose}

treatment. Principal coordinates analysis (PCoA) of UniFrac distances for the cecal and fecal microbiota. The analysis was done by Weighted (relative abundance) or Unweighted (presence/absence) UniFrac for cecal or fecal microbiota, respectively. Mice were treated with $8 \%$ fructose water for 12 weeks. Feces were collected at 1, 2, 4, and 12 weeks. Cecum was collected at experiment completion at 12 weeks. Effects of strain are seen in cecum $(A ; n=16$ for each strain) and feces (B; $n=64$ for each strain), and the influence of time is observed in feces (C). Fecal microbiota of individual mouse strains was overlaid with time for B6 (D), DBA (E), and FVB (F), and treatment for B6 (G), DBA (H), and FVB (I). Samples within the dotted circles are from the 12-week time point, and the p-values refer to the differences between treatments at this time point. Cecal microbiota of B6 $(J, n=16)$, DBA $(K, n=16)$, and FVB $(L, n=16)$ mice were 
overlaid with treatment (fructose vs. water). Percent variance explained by axes is shown in parentheses. Different colors represent different mouse strains, time points, and treatment groups. Categorical groups were confirmed to have similar multivariate homogeneity of group dispersions to allow them to be compared using the non-parametric PERMANOVA test to report significance $\mathrm{p}$ values for grouping with each variable. If dispersions were different, PERMANOVA was not used and no test value was reported $(\mathrm{p}=\mathrm{NA})$.

Figure 3. Differences in the baseline microbial composition and correlation between relative abundance and adiposity gain across strains. Relative abundance of baseline fecal microbiota was compared between B6, DBA, and FVB mouse strains. The baseline microbiota levels were analyzed using the microbiota from mice of the water group of all time points. (A) Lactobacillus, (B) Clostridiales, (C) Lachnospiraceae, (D) S24-7, (E) Akkermansia, (F) Turicibacrer. Each bar represents an individual mouse sample ( $\mathrm{n}=30-32$ for each mouse strain). The black line represents the mean, and the dotted line represents the median. Relative abundance of baseline microbiota was correlated with adiposity gain upon fructose treatment for 12 weeks (G-K, $\mathrm{n}=7-8 /$ mouse strain). $\mathrm{r}=$ Biweight midcorrelation coefficient, $\mathrm{p}=$ BenjaminiHochberg adjusted p-values.

\section{Figure 4. Correlation between fructose-responsive fecal microbiota, unknown genus of} Rikenellaceae in DBA mice and metabolic phenotypes. The relative abundance of unknown genus of Rikenellaceae in DBA mice was correlated with metabolic phenotypes. Data were collected for both water and fructose group at 1, 2, 4, and 12 weeks of fructose treatment. Water (top) group and fructose (bottom) group were analyzed separately ( $\mathrm{n}=7-8 /$ group/time point) 
across all time points for body weight (A), adiposity (B), and $\mathrm{AUC}_{\text {glucose }}(\mathrm{C})$. $\mathrm{r}$ stands for Biweight midcorrelation coefficient; $\mathrm{p}$ indicates Benjamini-Hochberg adjusted p-values.

Figure 5. Effects of B6 microbiota on fructose-induced metabolic phenotypes in DBA mice. Schematic design of fecal transplant (A). Gut microbiota was first cleared by antibiotics treatment, and then donor fecal supernatant from B6 was transplanted to the recipient DBA mice by oral gavage, and vice versa. B6 mice that received DBA feces were designated as B6(DBA), and DBA mice that received B6 feces were labeled as DBA(B6). After 1 week of fecal transplant, B6(DBA) or DBA(B6) mice were divided to two groups (fructose and water group), and challenged by $8 \%$ fructose water or drinking water for 12 weeks. Metabolic phenotypes of body weight $(\mathrm{B}, \mathrm{C})$, fat mass (D), and glucose level during IPGTT (E), were collected. NS stands for nonsignificant by Student's t-test. Data are presented as mean \pm SEM; $\mathrm{n}=8$ except B6 (DBA) water group $(\mathrm{n}=5)$.

\section{Figure 6. Effects of Akkermansia inoculation to DBA mice on fructose-induced metabolic}

phenotypes. Schematic design of Akkermansia inoculation (A). Gut microbiota were first cleared by antibiotics treatment, and then anaerobically cultured A. muciniphila was orally gavaged to the recipient DBA mice, labeled as DBA(Akk). After 1 week of microbial gavage, DBA(Akk) mice were divided to two groups (fructose and water group), and challenged by $8 \%$ fructose water or drinking water for 8 weeks. Body weight (B), fat mass (C), and glucose level during IPGTT (D) were measured. NS stands for nonsignificant by Student's t test. Data are mean \pm SEM; $n=6$ for water group and $n=7$ for fructose group. 
Figure 1

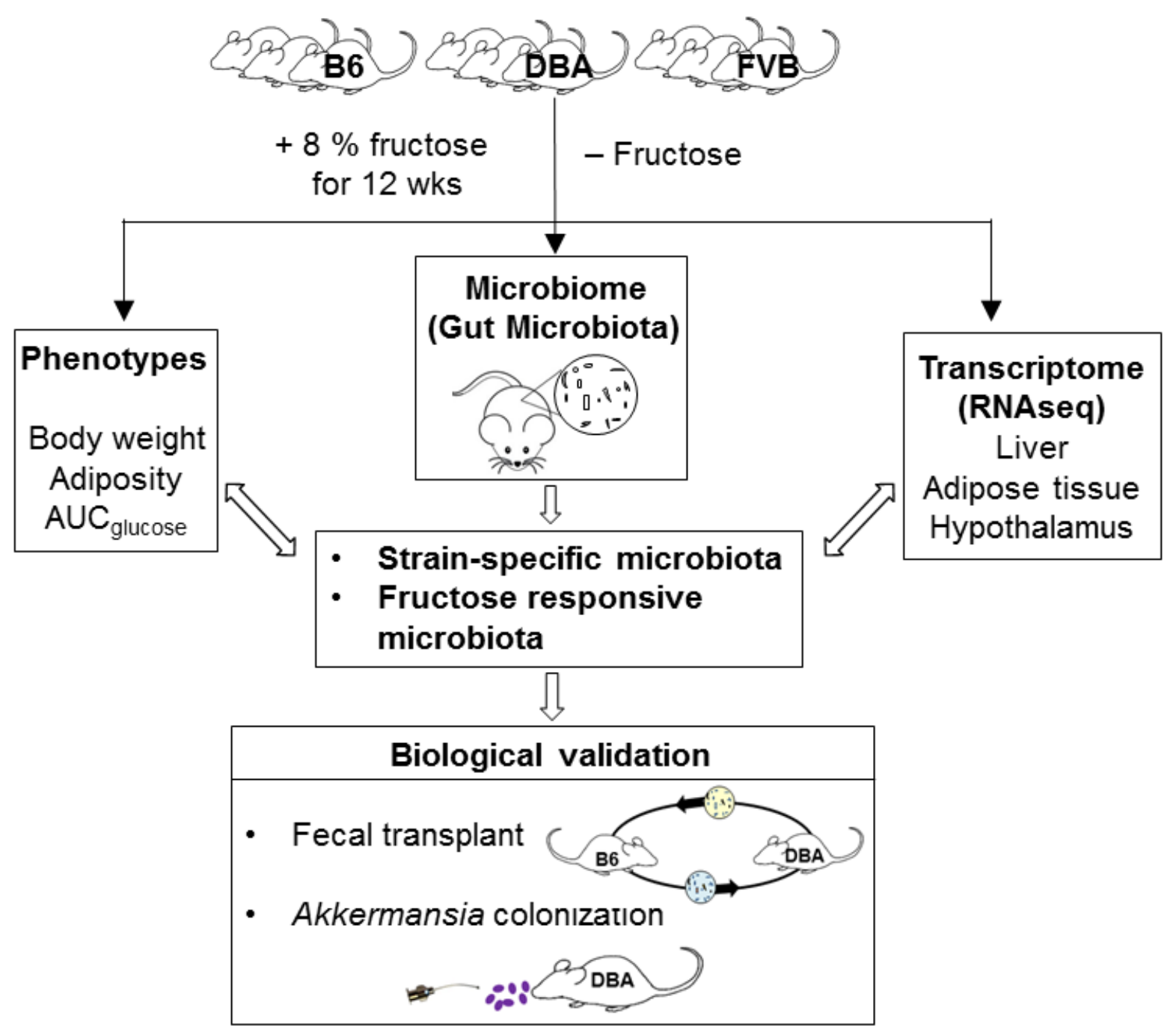


Figure 2
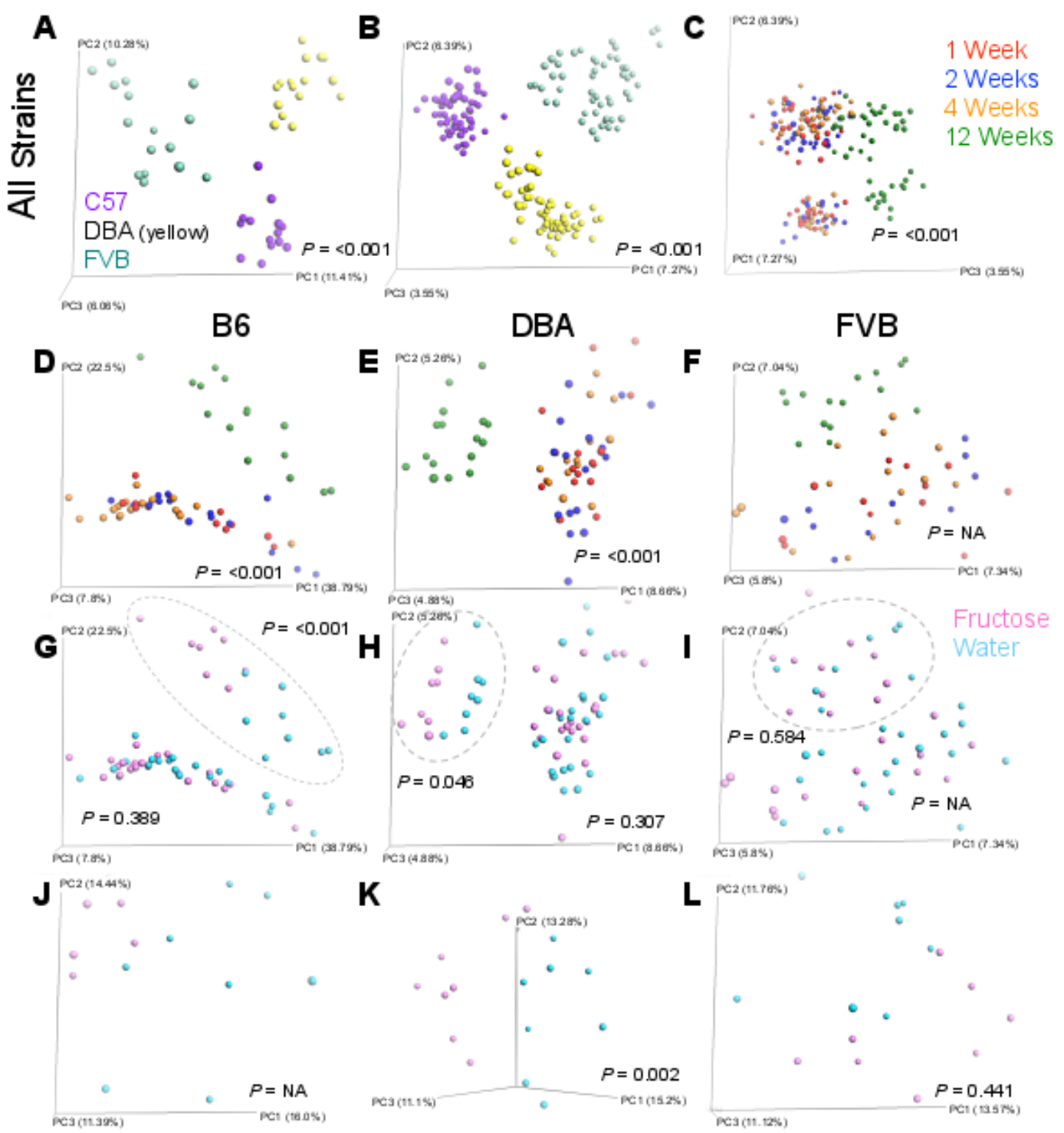
Figure 3
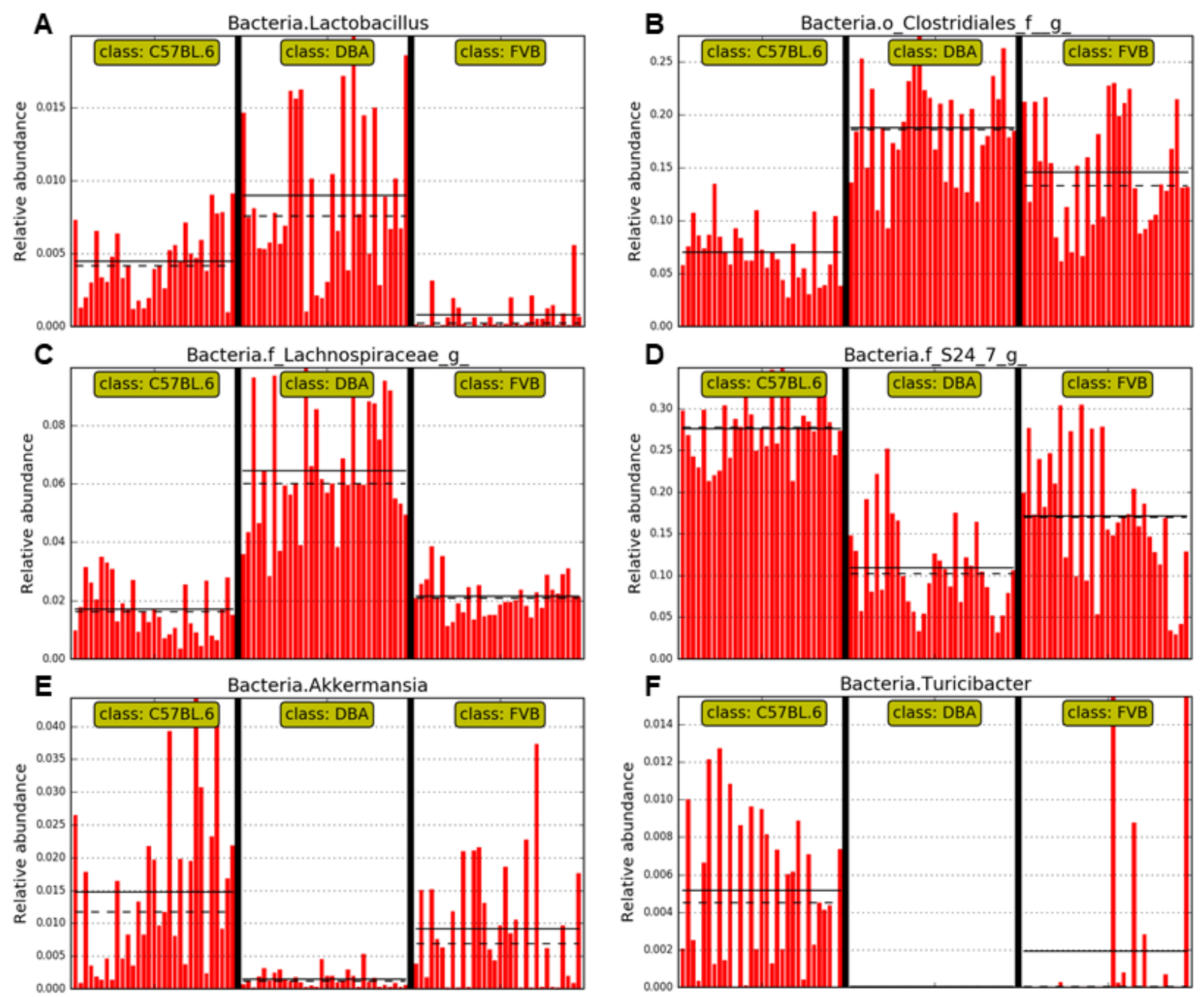

G

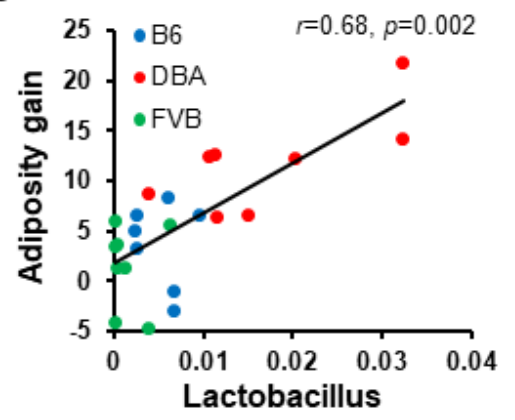

$J$

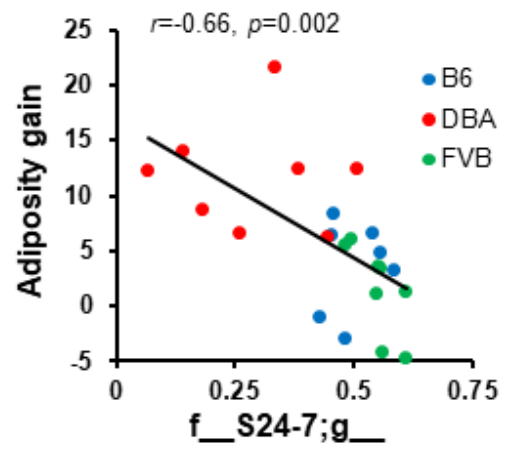

H

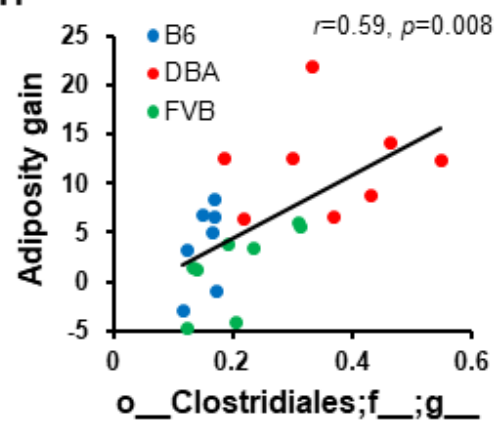

K

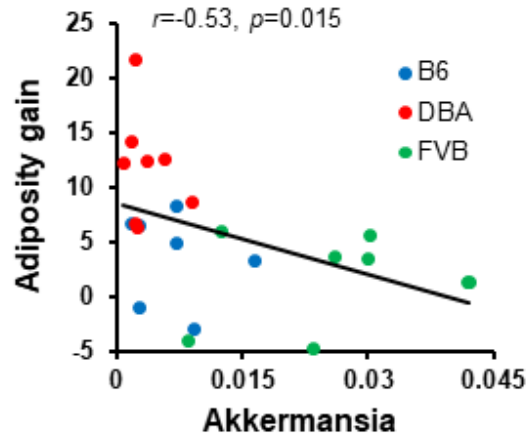

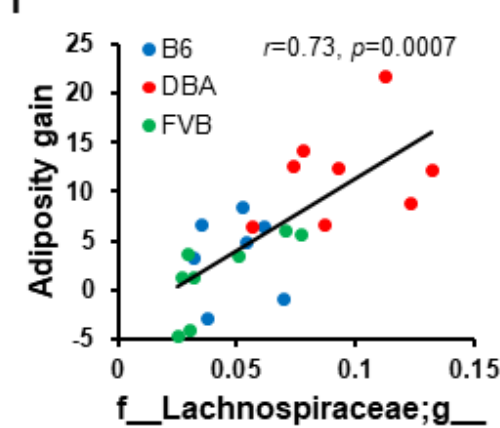




\section{Figure 4}

A
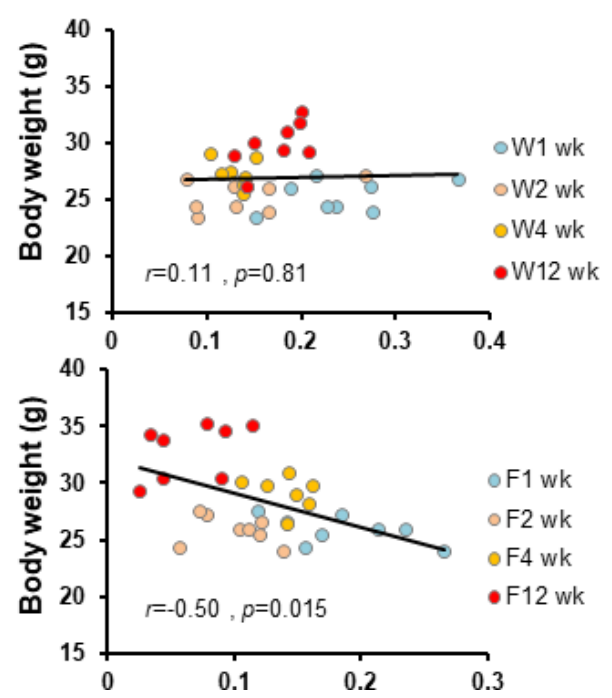

B

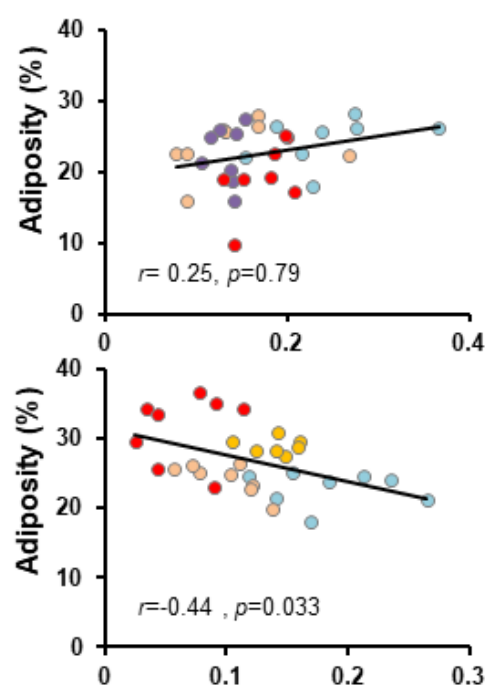

C

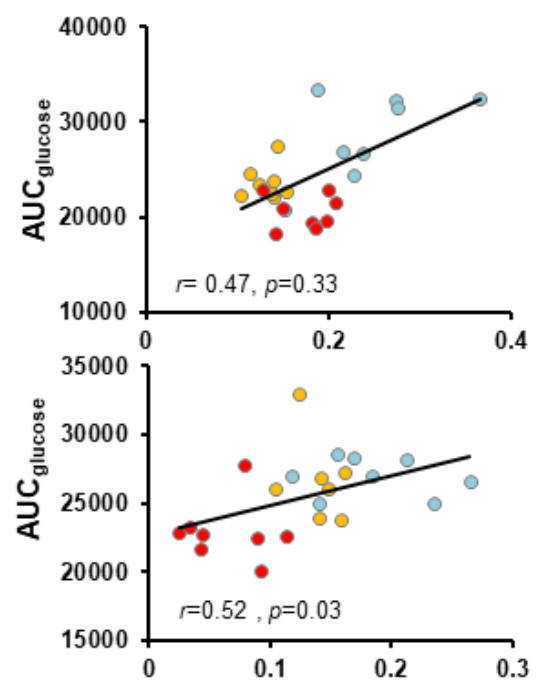




\section{Figure 5}

A

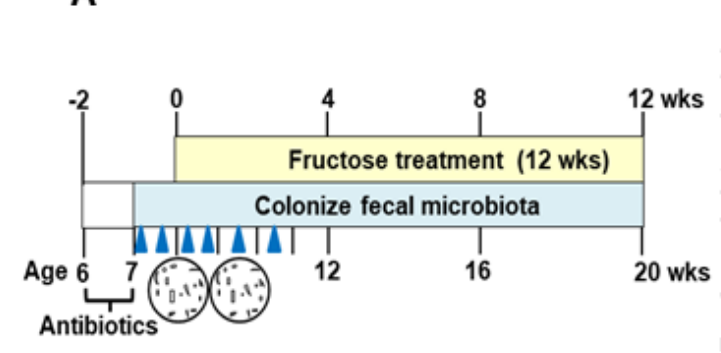

D

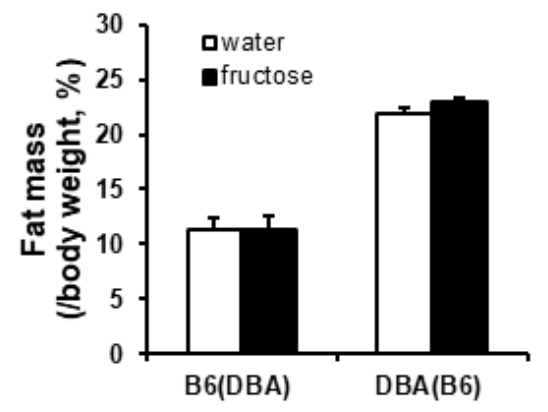

B

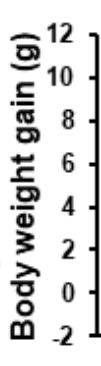

0
B6(DBA) mice

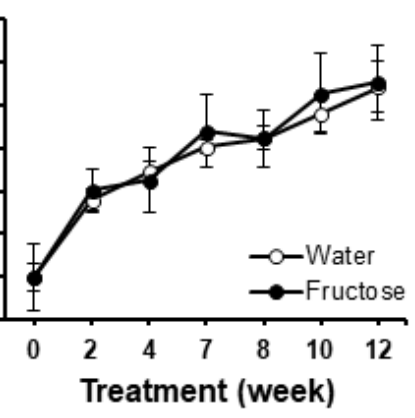

C

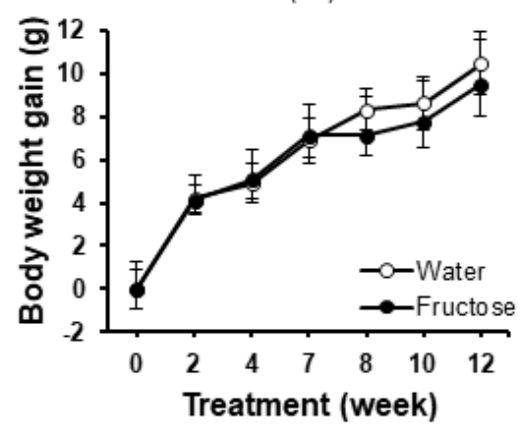

$\mathbf{E}$

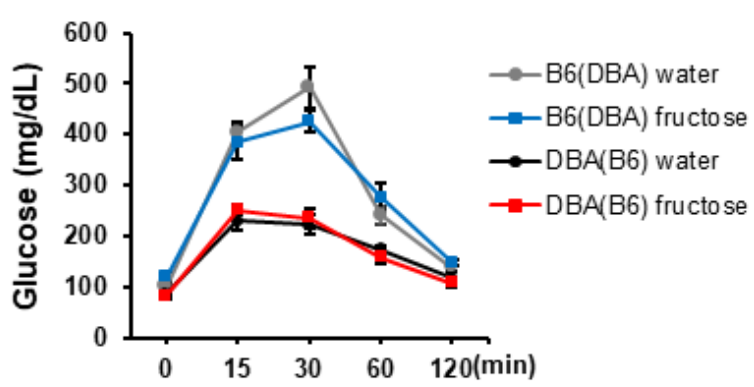




\section{Figure 6}

A

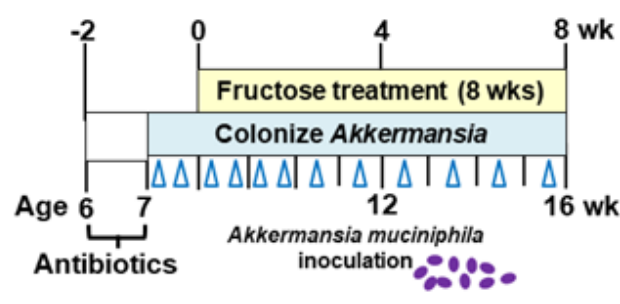

C

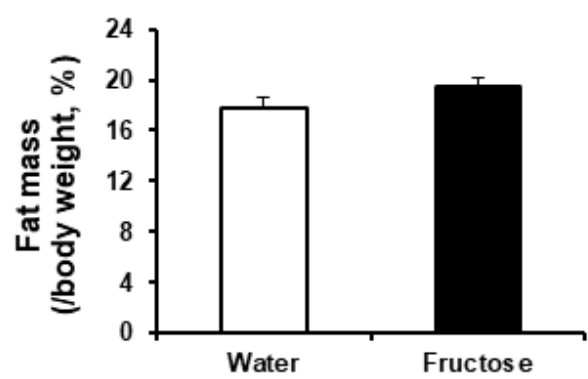

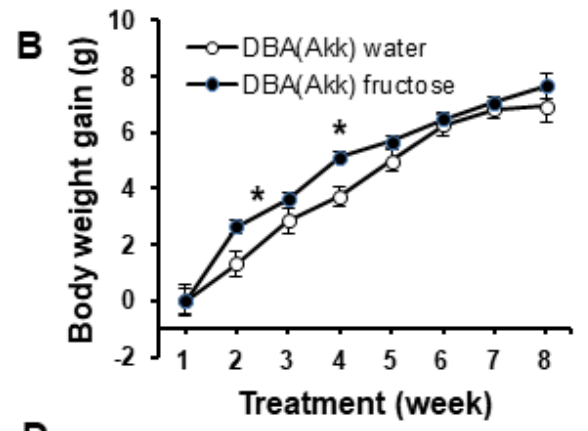

D

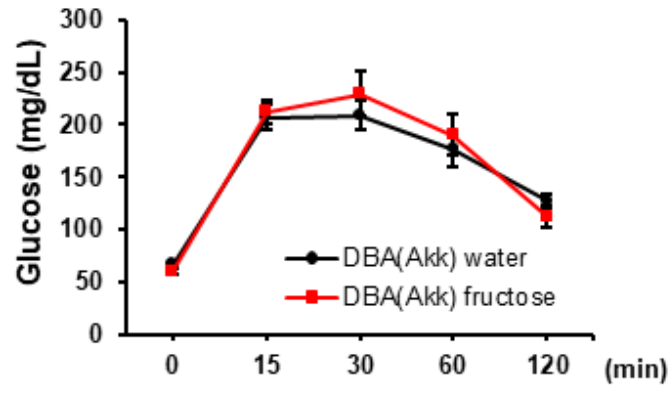

